\title{
Predicting perioperative mortality after radical cystectomy: comorbidity assessment tools are only part of the puzzle
}

\author{
Valérie Fonteyne $^{1}$, Elke Rammant ${ }^{1}$, Karel Decaestecker ${ }^{2}$ \\ ${ }^{1}$ Department of Radiotherapy Oncology, ${ }^{2}$ Department of Urology, Ghent University Hospital, Ghent, Belgium \\ Correspondence to: Valérie Fonteyne. Department of Radiation Oncology, Ghent University Hospital, Corneel Heymanslaan 10, 9000 Ghent, Belgium. \\ Email: Valerie.fonteyne@uzgent.be. \\ Provenance: This is an invited article commissioned by Section Editor Xiao Li (Department of Urology, Jiangsu Cancer Hospital \& Jiangsu Institute \\ of Cancer Research \& Nanjing Medical University Affiliated Cancer Hospital, Nanjing, China). \\ Comment on: Dell'Oglio P, Tian Z, Leyh-Bannurah SR, et al. Development of a New Comorbidity Assessment Tool for Specific Prediction of \\ Perioperative Mortality in Contemporary Patients Treated with Radical Cystectomy. Ann Surg Oncol 2019;26:1942-9.
}

Submitted Jun 29, 2019. Accepted for publication Jul 09, 2019.

doi: $10.21037 /$ tau.2019.07.06

View this article at: http://dx.doi.org/10.21037/tau.2019.07.06

A radical cystectomy $(\mathrm{RC})$ is the mainstay of treatment for patients with muscle invasive bladder cancer (MIBC) (1). Despite improvements in surgical techniques and peri-operative management, the incidence of complications after RC remains significant. Even in contemporary series the incidence of grade 3-5 complications within 90 days after RC is high and occurs in up to $19 \%$ of the patients (2). The reported 90 -day mortality rate varies between $1.2 \%$ and $8 \%(3,4)$.

Optimal patient selection for radical surgery is imperative in order to improve the outcome. The European guidelines of urology for MIBC do recommend routine assessment of comorbidity by validated scores such as the age-adjusted Charlson comorbidity index (CCI), which is without any doubt the most examined one for MIBC (1). In the setting of MIBC, CCI is found to be correlated with postoperative, cancer-specific and overall mortality $(5,6)$. However, and as pointed out in the article by Dell'Oglio et al. most of the current comorbidity indices were not specifically designed for patients with MIBC undergoing RC (7). Patients with MIBC however do represent a more fragile group of patients as patients often have multiple comorbidities making them more vulnerable for therapyinduced toxicity. To encounter this problem Dell'Oglio et al. developed a comorbidity assessment tool for predicting 90 days-mortality after RC solely based on data charts of MIBC patients (7). Dell'Oglio et al. retained 7 comorbid conditions (diabetes, osteo-arthrose, congestive heart failure, chronic pulmonary disease, anemia, cardiomegaly, aortocoronary bypass) and 2 disease manifestations (urinary tract infection and hydronephrosis) that predicted for 90-day mortality (7). The majority of these predictors are closely linked to the most reported causes of early death after RC as described in literature being septicaemia, heart failure, pneumonia as well as genitourinary, gastrointestinal or haematological complications (8).

In contrast to the widely applied age adjusted CCI, the model of Dell'Oglio et al. does not include patient's age (7). It has been recognized that physiological age rather then chronological age should be taken into account and age on itself does not preclude patients from curative treatments such as RC (9).

In the meanwhile novel patient-specific risk factors have been defined, which were also not included in the model proposed by Dell'Oglio et al. Although based on retrospective analysis, an association with post-RC morbidity was reported for frailty score, nutritional status and sarcopenia $(2,10)$.

Multiple tools exist to measure frailty. The Fried Frailty Criteria, comprising 5 assessments, was evaluated in a large prospective trial examining frailty and RC (2). Burg et al. found that Fried Frailty Criteria were predictive for highgrade complications (2). As both the incidence of MIBC as well as the incidence of frailty increases with age, frailty assessment should be considered before taking treatment decisions.

The pathogenesis of malnutrition is multifactorial and 
involves tumour-derived factors, host-derived factors inducing metabolic derangements as well as side effects of anticancer therapies (11). Malnutrition will ultimately lead to muscle waist and is associated with pulmonary complications and impaired immune responses that increase the susceptibility to infections. Lack of awareness of the nutritional status of cancer patients results in further weight loss which is often undiagnosed until it becomes severe. A recent meta-analysis confirmed the negative impact of malnutrition on mortality specifically among older patients with cancer (12). As malnutrition is a modifiable factor, much interest goes to the implementation of nutritional interventions with the aim to improve the outcome of the patients. The positive effect of it has been shown in the setting of compliance of gastric cancer patients undergoing chemotherapy and was recently also evaluated within a Cochrane systematic review for patients with MIBC exclusively (13). Despite the paucity of literature and based on low-quality evidence the author's concluded that immune-enhancing nutrition and oral nutrition may reduce complication rates. For sure, more prospective trials are needed to evaluate the true value of nutritional interventions (13).

However, also nutritional interventions should not be implemented in isolation of other supportive care interventions (e.g., exercise, psychosocial counselling) (14). In order to optimize the preoperative condition of the patients and consequently minimise postoperative morbidity, a multimodal prehabilitation approach should be used (14). Prehabilitation programs aim to reduce risks and complications of planned procedures by optimizing the physical and psychosocial condition of the patients (14).

In patients with MIBC, preoperative functional status (15) and mental health (16) have been associated with risk of morbidity and mortality following RC.

Also, evidence in other cancer populations showed benefits of (preoperative) exercise on postoperative pulmonary complications (17), overall postoperative morbidity (18) and even survival (19).

Although evidence in bladder cancer is scarce, preoperative exercise training already showed benefits for muscle strength and physical fitness (19). However, more evidence in MIBC is needed to evaluate the effects of preoperative exercise on postoperative complications and mortality (19). Another potential area for making the patient 'fit for surgery' is related to the mental wellbeing of the patients. The diagnosis and treatment of cancer causes psychological distress in patients undergoing major surgery, which might influence postoperative outcomes. Although again more research is needed in this area, evidence suggests positive effects of psychological interventions on immunological function and patient-reported psychological outcomes, quality of life, and somatic symptoms (20). More focus on psychological optimization might especially be important for patients with MIBC.

Despite the usual focus on unimodal interventions, it is important to recognize the potential contribution and synergistic effects of one intervention on the others (14).

Enhanced recovery after surgery (ERAS) protocols involve a multimodal approach including interventions at all stages of care (e.g., preoperative counselling, optimization of nutrition, standardized analgesic and anesthetic regimens, early mobilization).

ERAS protocols have also been developed to encounter differences in peri-operative management of patients undergoing major surgery. Within the setting of MIBC, ERAS protocols reduce length of hospital-stay, time-tobowel function and rate of complications after cystectomy. So far however there are no data yet indicating that the implementation of ERAS protocols results in significant decrease of 90-day mortality (21).

Apart from patient and tumor characteristics, postoperative mortality is affected by multiple other factors.

So has the impact of hospital volume on postoperative outcomes been proven specifically in the era of gastrointestinal oncology as well as elderly patients, advocating centralization of care for these patients. A recent assessment in readmission and mortality rate after surgery for cancer, including bladder cancer, confirmed that there are substantial between-hospital variations. So was a higher mean annual surgical volume associated with a decreased inhospital mortality $(4,22)$.

Robot-assisted RC (RARC) has gained popularity as it has been hypothesised that, in comparison with open $\mathrm{RC}, \mathrm{RARC}$ results in less blood loss, shorter recovery time and less complications with comparable oncological outcome. Based on 5 randomised trials comparing open RC with RARC, Rai et al. concluded that both modalities have similar outcomes with regard to time to recurrence, rates of major complications, quality of life and positive margin rates (23). Whether RARC reduces rates of minor complications is doubtful although it reduces the risk of blood transfusions and hospital stay. However, in all reported randomized trials to date, RARC has always been combined with extracorporeal urinary diversion. Whether optimal RARC with intracorporeal urinary diversion is able 
to reduce complication rates, is now tested in the phase 3 randomized iROC trial (NCT03049410).

Finally, long term results of trimodality therapy (TMT) as a bladder sparing alternative for RC show good oncological and functional outcome. Although large trials randomizing patients between RC and TMT are lacking, matched case analysis illustrate that the oncological results for both treatment options are comparable and the added value of a true multidisciplinary approach for MIBC patients has been clearly demonstrated in the publication by Kulkarni and colleagues (24). Having a valuable alternative for surgery for MIBC further empowers the importance of patient selection for aggressive surgery such as a RC.

In conclusion, the proposed model by Dell'Oglio represents a practical tool that can easily be implemented in daily practice to predict 90-day mortality (7). However, the tool should be used in the right context taking into account the study selection criteria (25). The tool was designed for patients aged 66 years and older and was developed based on ICD-9-CM codes. Prospective studies are required to evaluate whether this model also applies for diagnoses outside the ICD-9-CM format. As peri-operative mortality depends on several factors, better accuracy might be expected by more sophisticated modelling procedures also taking into account patient frailty and nutrition status as well as physician and hospital-specific data.

\section{Acknowledgments}

None.

\section{Footnote}

Conflicts of Interest: $\mathrm{V}$ Fonteyne is a post-doctorate clinical specialist funded by the Belgian Foundation against Cancer. The other authors have no conflicts of interest to declare.

Ethical Statement: The authors are accountable for all aspects of the work in ensuring that questions related to the accuracy or integrity of any part of the work are appropriately investigated and resolved.

\section{References}

1. Alfred Witjes J, Lebret T, Compérat EM, et al. Updated 2016 EAU Guidelines on Muscle-invasive and metastatic bladder cancer. Eur Urol 2017;71:462-75.

2. Burg ML, Clifford TG, Bazargani ST, et al. Frailty as a predictor of complications after radical cystectomy: a prospective study of various preoperative assessments. Urol Oncol 2019;37:40-7.

3. Gilbert SM, Dunn RL, Miller DC, et al. Mortality after urologic cancer surgery: impact of non-index case volume. Urology 2008;71:906-10.

4. Porter MP, Gore JL, Wright JL. Hospital volume and 90day mortality risk after radical cystectomy: a populationbased cohort study. World J Urol 2011;29:73-7.

5. Koppie TM, Serio AM, Vickers AJ, et al. Ageadjusted Charlson comorbidity score is associated with treatment decisions and clinical outcomes for patients undergoing radical cystectomy for bladder cancer. Cancer 2008;112:2384-92.

6. Abdollah F, Sun M, Schmitges J, et al. Development and validation of a reference table for prediction of postoperative mortality rate in patients treated with radical cystectomy: a population-based study. Ann Surg Oncol 2012;19:309-17.

7. Dell'Oglio P, Tian Z, Leyh-Bannurah SR, et al. Development of a new comorbidity assessment tool for specific prediction of perioperative mortality in contemporary patients treated with radical cystectomy. Ann Surg Oncol 2019;26:1942-9.

8. Takada N, Abe T, Shinohara N, et al. Peri-operative morbidity and mortality related to radical cystectomy: a multi-institutional retrospective study in Japan. BJU Int 2012;110:E756-64.

9. Fonteyne V, Ost P, Bellmunt J, et al. Curative treatment for muscle invasive bladder cancer in elderly patients: a systematic review. Eur Urol 2018;73:40-50.

10. Mayr R, Fritsche HM, Zeman F, et al. Sarcopenia predicts 90-day mortality and postoperative complications after radical cystectomy for bladder cancer. World J Urol 2018;36:1201-7.

11. Muscaritoli M, Molfino A, Gioia G, et al. The 'parallel pathway': a novel nutritional and metabolic approach to cancer patients. Intern Emerg Med 2011;6:105-12.

12. Zhang X, Tang T, Pang L, et al. Malnutrition and overall survival in older adults with cancer: a systematic review and meta-analysis. J Geriatr Oncol 2019. doi: 10.1016/ j.jgo.2019.03.002.

13. Burden S, Bilson HA, Lal S, et al. Perioperative nutrition for the treatment of bladder cancer by radical cystectomy. Cochrane Database Syst Rev 2019;5:CD010127.

14. Scheede-Bergdahl C, Minnella EM, Carli F. Multi-modal prehabilitation: addressing the why, when, what, how, who and where next? Anaesthesia 2019;74:20-6. 
15. Jensen BT, Laustsen S, Jensen JB, et al. Exercise-based prehabilitation is feasible and effective in radical cystectomy pathways - secondary results from a randomized controlled trial. Support Care Cancer 2016;24:3325-31.

16. Sharma P, Henriksen CH, Zargar-Shoshtari K, et al. Preoperative patient reported mental health is associated with high grade complications after radical cystectomy. J Urol 2016;195:47-52.

17. Piraux E, Caty G, Reychler G. Effects of preoperative combined aerobic and resistance exercise training in cancer patients undergoing tumour resection surgery: A systematic review of randomised trials. Surg Oncol 2018;27:584-94.

18. Cormie P, Zopf EM, Zhang X, et al. The impact of exercise on cancer mortality, recurrence, and treatmentrelated adverse effects. Epidemiol Rev 2017;39:71-92.

19. Rammant E, Decaestecker K, Bultijnck R. A systematic review of exercise and psychosocial rehabilitation interventions to improve health-related outcomes in patients with bladder cancer undergoing radical cystectomy. Clin Rehabil 2018;32:594-606.

20. Tsimopoulou I, Pasquali S, Howard R, et al. Psychological prehabilitation before cancer surgery: a systematic review. Ann Surg Oncol 2015;22:4117-23.

21. Altobelli E, Buscarini M, Gill H, et al. Readmission rate and causes at 90-day after radical cystectomy in patients on early recovery after surgery protocol. Bladder Cancer 2017;3:51-6.

22. Haneuse S, Dominici F, Normand S, et al. Assessment of between-hospital variation in readmission and mortality after cancer surgical procedures. JAMA Network Open 2018; 1:e183038.

23. Rai BP, Bondad J, Vasdez N, et al. Robotic versus open radical cystectomy for bladder cancer in adults. Cochrane Database Syst Rev 2019;4:CD011903.

24. Kulkarni GS, Hermanns T, Wei Y, et al. Propensity score analysis of radical cystectomy versus bladder sparing trimodal therapy in the setting of a multidisciplinary bladder cancer clinic. J Clin Oncol 2017;35:2299-305.

25. Shariat SF, Karakiewicz PI, Suardi N, et al. Comparison of nomograms with other methods for predicting outcomes in prostate cancer: a critical analysis of the literature. Clin Cancer Res 2008;14:4400-7.
Cite this article as: Fonteyne V, Rammant E, Decaestecker K. Predicting perioperative mortality after radical cystectomy: comorbidity assessment tools are only part of the puzzle. Transl Androl Urol 2019;8(6):781-784. doi: 10.21037/tau.2019.07.06 